\title{
Screening and Tunneling at Metal Surfaces*
}

\author{
V. E. Kenner, R. E. Allen, and W. M. Saslow \\ Department of Physics, Texas A\&M University, College Station, Texas 77843
}

(Received 15 November 1972)

\begin{abstract}
The screening of an external electric field near a metal surface has been determined for the semi-infinite free-electron model with the use of a self-consistent linear-response theory. The resulting self-consistent potential has been used to calculate tunneling (i.e., field-emission) currents.
\end{abstract}

\section{INTRODUCTION}

One of the best-known properties of a conductor is that the free charge can distribute itself near the surface so as to shield the interior from an external electric field. In classical electrostatics, the charge induced by the electric field is located exactly at the surface, and the electric field drops abruptly to zero inside the metal. Quantum effects give a finite penetration depth for the external field and a finite thickness for the charge distribution near the surface. This penetration of the applied field is of interest in regard to, e. g. , gravitationally induced electric fields ${ }^{1,2}$ and field emission. ${ }^{3-5}$

A key consideration in calculations of the screened field is self-consistency. Non-self-consistent potential barriers have long been used in field-emission calculations, beginning with the triangular barrier used by Fowler and Nordheim in $1928 .^{6}$ Later an image term was added, ${ }^{7,8}$ and other barrier shapes have since been used. ${ }^{9} \mathrm{Re}-$ cently Theophilou and Modinos ${ }^{3}$ studied field penetration and field emission in a "semi-self-consistent" model. Their procedure was to assume a parametrized form for the one-electron potential and then determine the parameters in such a way as to satisfy continuity and charge neutrality requirements, and to agree with the correct work function for tungsten. Although this procedure has advantages in calculating tunneling currents (see Sec. IV), a treatment which is not fully selfconsistent can lead to unphysical results. We mention, for example, the failure of the "field penetration" $x_{0}$ io go to zero as the applied electric field goes to zero (see Fig. 9 of Ref. 3). More generally, the induced charge density, the induced potential, and the screening of the electric field will contain contributions which are due to the inconsistency of the original potential (for zero electric field) as well as the presence of the field. We therefore consider it worthwhile to carry out a truly self-consistent calculation of the screening of an electric field, the distribution of excess charge, and the tunneling of electrons at a metal surface.
We will adopt the semi-infinite free-electron model, in which the ions in the metal are replaced by a uniform positive background which abruptly terminates at the surface. ${ }^{10}$ For this model, the quantum-mechanical screening of an external electric field can be calculated nearly self-consistently with a modified version of the linear-response theory developed by Schiff. ${ }^{1}$ Schiff's method requires the self-consistently determined wave functions in the absence of an applied field; these wave functions can be obtained from the work of Lang and Kohn. ${ }^{10}$ The present authors, ${ }^{11}$ neglecting exchange and correlation contributions, have solved Schiff's linear-response equations self-consistently, starting from the zero-field results of Lang and Kohn. In the present paper, exchange and correlation are taken into account, in the approximation due to Wigner, ${ }^{12}$ for $r_{s}=2$ and $r_{s}=5$. The linear-response solution yields the screened external field and the potential barrier through which an electron must tunnel to produce an emission current.

In Sec. II we describe the method in more detail and give the results for the screened electric field. In Sec. III we discuss the equivalent problem of the distribution of excess charge near the surface and give our results (which are in agreement with those obtained by Lang and Kohn ${ }^{13}$ using a different method). In Sec. IV, we apply the results of Sec. II in determining field-emission currents due to the tunneling of electrons out of the metal when an electric field is applied. A brief summary of our results is given in Sec. V.

\section{SCREENING OF AN EXTERNAL ELECTRIC FIELD}

\section{A. Theory}

For the semi-infinite free-electron model the single-electron self-consistency problem with zero external field can be put in the form

$$
\begin{aligned}
& \left(-\frac{d^{2}}{d z^{2}}+v_{0}(z)\right) \psi_{k}^{(0)}(z)=k^{2} \psi_{k}^{(0)}(z), \\
& n_{0}(z)=\left(1 / \pi^{2}\right) \int_{0}^{k_{F}}\left(k_{F}^{2}-k^{2}\right) \psi_{k}^{(0)}(z)^{2} d k \\
& v_{0}(z)=v_{\mathrm{os}}^{(0)}(z)+v_{\mathrm{xc}}^{(0)}(z) .
\end{aligned}
$$


[We use atomic units so that $\hbar=e=m=1$. Note that a factor of 2 is absorbed in $v_{0}(z)$.] Here $z$ is the coordinate normal to the surface, $k=k_{z}, k_{F}$ is the Fermi momentum, $n_{0}(z)$ is the electronic number density, $v_{0}(z)$ is the effective potential which goes to zero inside the metal and to a constant value outside, and $v_{\text {os }}^{(0)}(z)$ is the electrostatic part of $v_{0}(z) . \quad \psi_{k}^{(0)}$ is the electronic wave function, which goes to zero as $z-+\infty$ and to $\sin \left(k z+\alpha_{k}\right)$ as $z \rightarrow-\infty$. We have adopted the usual assumption that exchange and correlation effects can be approximately taken into account with the use of a local potential $v_{\mathrm{xc}}^{(0)}(z)$. We follow Ref. 10 in adopting the expression of Wigner ${ }^{12}$ for this potential.

Equations (1) are the equations which Lang and Kohn ${ }^{10}$ have solved self-consistently with no external field. The presence of an applied field perturbs the effective potential, thereby altering the electronic wave functions and changing the electronic number density. We define the altered quantities $v(z), \psi_{k}(z)$, and $n(z)$ by

$$
\begin{aligned}
& v(z)=v_{0}(z)+\delta v(z), \\
& \psi_{k}(z)=\psi_{k}{ }^{(0)}(z)+\delta \psi_{k}(z), \\
& n(z)=n_{0}(z)+\delta n(z),
\end{aligned}
$$

where $\delta v(z)=\delta v_{\text {es }}(z)+\delta v_{x c}(z), \delta \psi_{k}(z)$, and $\delta n(z)$ are, respectively, the changes in the effective potential, an electronic wave function, and the electronic density due to application of the electric field. These altered quantities must satisfy Eqs. (1) with their appropriate boundary conditions, as did $v_{0}$, $\psi_{k}^{(0)}$, and $n_{0}$. The boundary condition on $v(z)$ external to the metal is now $d v_{0}(z) / d z \rightarrow 2 E_{0}$ as $z \rightarrow \infty$, where $E_{0}$ is the applied field.

Solving the self-consistency equations (1) to first order in the external field gives ${ }^{1}$

$$
\begin{aligned}
& \frac{d^{2}}{d z^{2}} \delta v_{\text {es }}(z)=-8 \pi \delta n(z), \\
& \delta n(z)=\left(2 / \pi^{2}\right) \int_{-\infty}^{\infty} F\left(z, z^{\prime}\right) \delta v\left(z^{\prime}\right) d z^{\prime}, \\
& F\left(z, z^{\prime}\right)=\int_{0}^{k F}\left(k_{F}^{2}-k^{2}\right) \psi_{k}^{(0)}(z) \psi_{k}^{(0)}(z) \psi_{k}^{(0)}\left(z^{\prime}\right)^{2} \frac{d k}{k} .
\end{aligned}
$$

Here $\bar{\psi}_{k}^{(0)}(z)$ is the solution of Eq. (1a) that is irregular at $+\infty$ and approaches $\cos \left(k z+\alpha_{k}\right)$ as $z \rightarrow-\infty$. It is essential that the $\psi_{k}^{(0)}(z)$ be selfconsistent solutions of Eqs. (1); otherwise, Eq. $(3 b)$ is invalid.

If changes due to exchange and correlation are neglected, $\delta v(z) \rightarrow \delta v_{\text {es }}(z)$ in Eq. (3b), and the equation iterated once by Schiff $^{1}$ results:

$$
\frac{d^{2} \delta v_{\text {es }}(z)}{d z^{2}}=\frac{-16}{\pi} \int_{-\infty}^{\infty} F\left(z, z^{\prime}\right) \delta v_{\text {es }}\left(z^{\prime}\right) d z^{\prime} .
$$

The present authors, ${ }^{11}$ attempting to solve Eq. (4) by the iterative method with $F\left(z, z^{\prime}\right)$ determined by the self-consistent results of Lang and Kohn, ${ }^{10}$ obtained after one iteration results comparable to those of Schiff inside the metal. However, outside the metal the normalized field $E / E_{0}$ did not approach unity. Furthermore, when more than one iteration was attempted it was found that the iterative method failed to converge; therefore, Eq. (4) must be solved by other means (see Sec. II B).

The portion of $\delta v(z)$ due to exchange and correlation omitted in Eq. (4) can be included approximately by expanding about the ground-state electronic density

$$
\begin{aligned}
\delta v_{\mathrm{xc}}(z) & \approx\left[\frac{d v_{\mathrm{xc}}(n(z))}{d n(z)}\right]_{n_{0}} \delta n(z) \\
& =-\frac{1}{8 \pi}\left[\frac{d v_{\mathrm{xc}}(n)}{d n}\right]_{n_{0}} \frac{d^{2} \delta v_{\mathrm{gs}}(z)}{d z^{2}},
\end{aligned}
$$

where the subscript $n_{0}$ indicates that the quantity in brackets is to be evaluated with $n(z)=n_{0}(z)$.

Equations (3a), (3b), and (5) can be used to obtain, after integration by parts,

$$
\begin{aligned}
& E(z)=\lambda \int_{-\infty}^{\infty} d z^{\prime} \varkappa\left(z, z^{\prime}\right) E\left(z^{\prime}\right), \\
& \lambda=-16 / \pi, \\
& \varkappa\left(z, z^{\prime}\right)=K\left(z, z^{\prime}\right)-\frac{1}{8 \pi} \frac{d}{d z^{\prime}}\left[\left(\frac{d v_{x c}(n)}{d n}\right)_{n_{0}} \frac{d}{d z^{\prime}} K\left(z, z^{\prime}\right)\right],
\end{aligned}
$$

$K\left(z, z^{\prime}\right)=\int_{-\infty}^{z} d z_{1} \int_{\alpha^{\infty}}^{\infty} d z_{2} F\left(z_{1}, z_{2}\right)$.

The kernel $\varkappa\left(z, z^{\prime}\right)$ is not symmetrical in $z$ and $z^{\prime}$. [Contrary to an assertion by Schiff, ${ }^{1} K\left(z, z^{\prime}\right)$ is also not symmetrical. ] $E(z)$ is the electric field as a function of $z$ : as $z \rightarrow+\infty, E(z) \rightarrow E_{0}$; as $z--\infty$, $E(z) \rightarrow 0$.

\section{B. Numerical Results}

In order to obtain a numerical solution for the electric field, we approximate the integral Eq. (6a) by the set of $N$ algebraic equations

$$
\begin{aligned}
& E\left(z_{i}\right)=\lambda \sum_{j=1}^{N} \overline{\mathcal{K}}\left(z_{i}, z_{j}\right) E\left(z_{j}\right), i=2, \ldots, N \\
& E\left(z_{N}\right)=E_{0}
\end{aligned}
$$

in the $N$ unknowns $E\left(z_{i}\right)$, with the points $z_{i}$ evenly spaced over a finite range and $z_{N}$ taken to be the point farthest outside the surface. Here $\bar{\kappa}\left(z_{i}, z_{j}\right)$ is the kernel $\boldsymbol{\kappa}\left(z_{i}, z_{j}\right)$ multiplied by appropriate weighting factors to approximate (6a) by (7a) according to Simpson's rule. The values

$$
\begin{array}{lll}
z_{1}=-10 \pi / 4 k_{F}, & z_{N}=\pi / k_{F}, & N=71 \text { for } r_{s}=5 \\
z_{1}=-3 \pi / k_{F}, & z_{N}=2 \pi / k_{F}, & N=101 \text { for } r_{s}=2
\end{array}
$$

were found to yield a solution for $E(z)$ which was accurate to within a few percent.

To solve Eqs. (7) we first must evaluate the ker- 
nel $\varkappa\left(z_{i}, z_{j}\right) . \quad K\left(z, z^{\prime}\right)$ can be evaluated directly using Eq. (6d), but the second term involving $\left[d v_{\mathrm{xc}}(n) / d n\right]_{n_{0}}$ must be treated carefully since the expansion (5) fails at low densities. However, at these low densities the electrostatic term is large, so that little is lost by neglecting $\delta v_{x c}(z)$ for sufficiently small $n_{0}$; we have taken $\delta v_{\mathrm{xc}}(z)=0$ for $n_{0}(z)<0.002 n_{0}(-\infty)$.

Results both with and without exchange and correlation are shown in Fig. 1 for $r_{s}=5$. The effect of exchange and correlation is to decrease the effectiveness of the screening, in the sense that the magnitude of $E(z)$ is generally increased for a fixed value of $z$. The explanation for this result (also obtained in Ref. 3) is obvious: According to Eqs. (2.8c) and (2.15a) of Ref. 10, the exchange and correlation potential tends to draw electrons into regions where the electronic density is large, so $\delta n(z)$ will be increased in those regions where it is already large and the amplitude of the Friedel oscillations in $\delta n(z)$ will be increased. The positions of the Friedel oscillations will, of course, be almost unaffected.

In Figs. 1-4 the long tick marks are at distances $\frac{1}{2} d, \frac{3}{2} d$, etc. , beneath the surface, where $d=(4 \pi / 3)^{1 / 3} r_{s}$ is the distance between two layers of ions in a hypothetical monovalent metal with a simple cubic structure and a (100) surface orientation. These marks thus provide a rough guide as to the location of the center of each layer of ions. The ion positions in a real metal will, of course, depend on the crystal structure and surface orientation. Figures 1 and 2 demonstrate that (i) the elec-

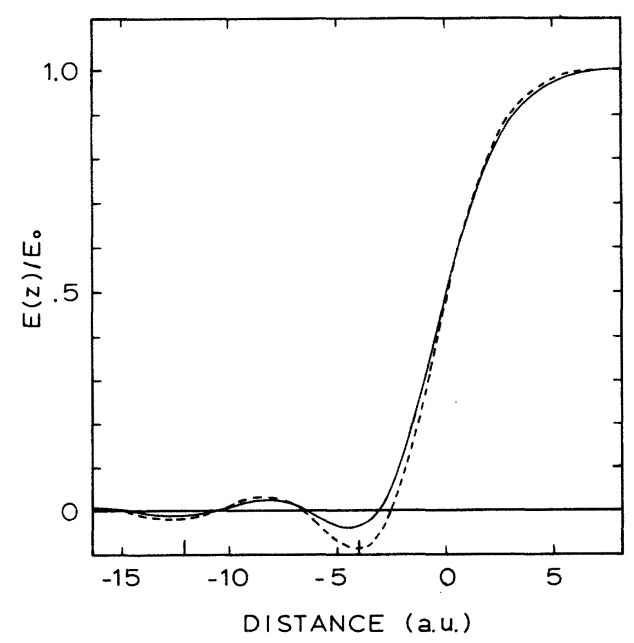

FIG. 1. Ratio of electric field $E(z)$ to external field $E_{0}$ for $r_{s}=5$. Solid line is without exchange and correlation; dashed line is with exchange and correlation. The long tick marks correspond to ion layers in a hypothetical monovalent metal with a simple cubic structure and a (100) surface orientation.

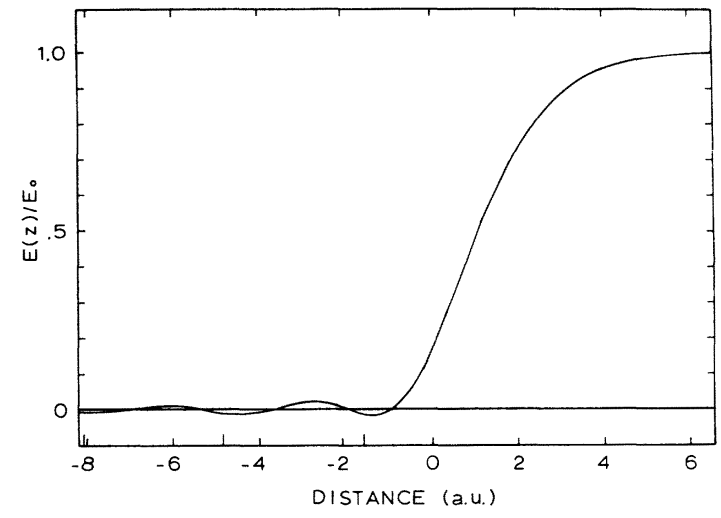

FIG. 2. Ratio of electric field $E(z)$ to external field $E_{0}$ for $r_{s}=2$. The long tick marks were determined as in Fig. 1. Exchange and correlation are included.

tric field is much smaller in amplitude at the center of the first layer of ions than it is outside the metal and (ii) the electric field can be "overscreened" at this point-i.e., $E$ is opposite in sign to its value outside the metal, so a test charge at this point would tend to move in the opposite direction compared to a test charge outside the metal. These results may be relevant to the problem of gravitationally induced electric fields. ${ }^{1}$

\section{DISTRIBUTION OF EXCESS CHARGE}

In classical electrostatics, if excess free charge is placed in a conductor, it will distribute itself exactly at the surface of the conductor. Quantum effects will lead to a distribution which has a finite thickness. The problem of the distribution of excess charge is equivalent to the screening problem of Sec. II: An external electric field $E_{0}$ will induce a surface charge density $\sigma=E_{0} / 4 \pi$ on one surface of a thick metal slab and a charge density $-\sigma$ on the other. Similarly, an excess charge density $\sigma$ on each surface of a thick slab will produce an electric field $E_{0}=4 \pi \sigma$ far from the slab on either side. For the semi-infinite free-electron model, we have in either case an excess surface charge density $\sigma=-e \int_{-\infty}^{\infty} \delta n(z) d z$ and an external electric field $E_{0}=E(+\infty)=4 \pi \sigma$. Gauss's law,

$$
\vec{\nabla} \cdot \overrightarrow{\mathrm{E}}(z)=\frac{d E(z)}{d z}=-4 \pi e \delta n(z),
$$

can be used to determine the excess electronic density $\delta n(z)$ from the screened field $E(z)$.

The distribution of excess charge obtained in this manner is shown in Figs. 3 and 4 for $r_{s}=5$ and 2, respectively. Results for $r_{s}=5$ are shown with exchange and correlation both neglected and included within the approximation of Eq. (5). The results with exchange and correlation are in excellent agreement with those obtained by Lang and 


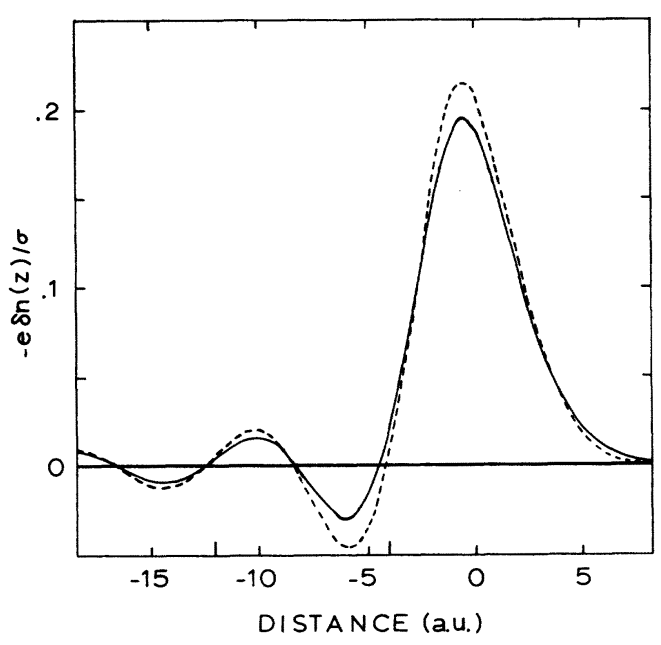

FIG. 3. Ratio of excess charge density $-e \delta n(z)$ to surface charge density $\sigma$ for $r_{s}=5$. Solid line is without exchange and correlation; dashed line is with exchange and correlation. The long tick marks were determined as in Fig. 1.

$\mathrm{Kohn}^{13}$ in their calculation of the work function. One interesting feature of the charge distribution is the shift of the peak in $\delta n(z)$ from inside the surface of the metal to outside as the $r_{s}$ value is changed from 5 to 2 .

We mention that all of the net excess charge lies outside of $z_{0}$, the point at which $E$ is first screened to zero in Figs. 1 and 2. This follows from integrating Gauss's law [Eq. (8)] and using $E\left(z_{0}\right)=0$, $E(+\infty)=4 \pi \sigma$ to get

$$
\int_{z_{0}}^{\infty}[-e \delta n(z)] d z=\sigma=\int_{-\infty}^{\infty}[-e \delta n(z)] d z .
$$

Within the context of linear-response theory, the point $z_{0}$ is independent of $E_{0}$.

\section{TUNNELING}

We now turn to the problem of field emission, in which the electrons tunnel out of the metal surface in the presence of an external field. Since strong fields must be used in order to obtain a measurable emission current, the applicability of linearresponse theory must first be considered. A measure of the size of nonlinear effects is provided by the size of the induced electronic density $\delta n(z)$. We find that for the strongest field that we will consider, $10^{8} \mathrm{~V} / \mathrm{cm}$, and at the peak in the induced density for $r_{s}=2$ [i.e., the largest value of $\delta n(z)$ in Fig. 4], the ratio $\delta n(z) / n_{0}(-\infty)$ is $1.6 \%$ and the ratio $\delta n(z) / n_{0}(z)$ is less than $7 \% .{ }^{14}$ Furthermore, for fields up to $10^{8} \mathrm{~V} / \mathrm{cm}$ the predicted nonlinear effects in Fig. 9 of Ref. 3 are smaller than effects due to the inconsistency of the zero-field potential. We therefore feel that linear-response theory will give a reasonable correction to the effective poten- tial even at rather strong fields.

A possibly more serious question concerns the zero-field potential. As is well known, tunneling probabilities involve the barrier height through an exponential factor. In the present case, the barrier height is given by the work function, so small errors in the work function will produce large errors in the tunneling probabilities and emission currents. Although the semi-infinite free-electron model yields moderately accurate values for the work functions of simple metals, ${ }^{13}$ the values are not exact, of course, and so our results will not be quantitatively correct if we carry out a calculation for a material with a given $r_{s}$ value. It may be more appropriate to regard our calculations of emission currents as being for self-consistent models with the specified work functions (3.89 and $2.73 \mathrm{eV}$, respectively) instead of metals with $r_{s}$ values of 2 and 5 . We mention that, although the method of Ref. 3 suffers from a lack of self-consistency, it has two advantages over our selfconsistent method in regard to the calculation of tunneling probabilities: The work function can be adjusted to the proper value for a given metal, and nonlinear effects are taken into account.

The potential barrier through which an electron tunnels can be determined from the results of Section II for the self-consistently screened electric field: If we let $V(z)$ be the effective one-electron potential energy, then

$$
\begin{aligned}
V(z)= & \frac{1}{2} v_{0}(z)+\frac{1}{2} \delta v_{x c}(z)+\frac{1}{2} \delta v_{0 s}(z), \\
=\frac{1}{2} v_{0}(z)+\frac{1}{2}\left[v _ { x c } \left(n_{0}(z)+\right.\right. & \left.\delta n(z))-v_{x c}\left(n_{0}(z)\right)\right] \\
& +\int_{-\infty}^{z} E\left(z^{\prime}\right) d z^{\prime},
\end{aligned}
$$

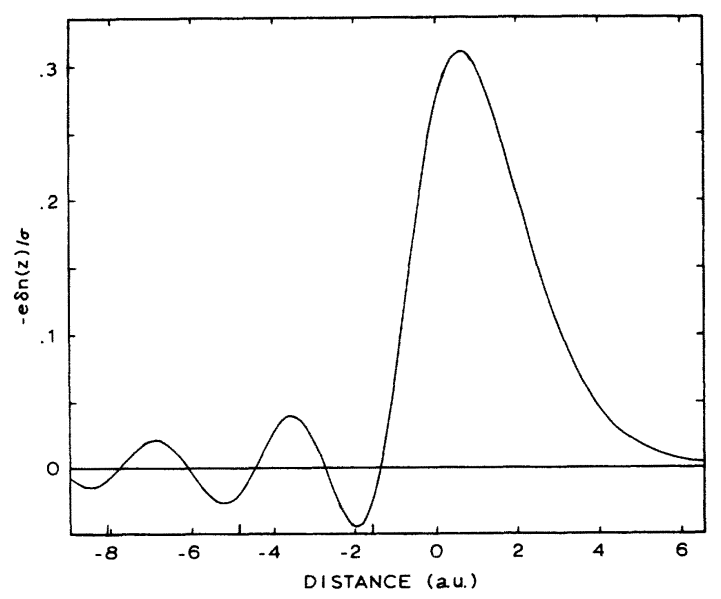

FIG. 4. Ratio of excess charge density $-e \delta n(z)$ to surface charge density $\sigma$ for $r_{s}=2$. The long tick marks were determined as in Fig. 1. Exchange and correlation are included. 
where the quantities on the right-hand side are defined in Sec. II. \{Recall that a factor of 2 was absorbed in $v_{0}, v_{\mathrm{xc}}$, and $v_{\mathrm{es}}$, and that $(d / d z)\left[\frac{1}{2} \delta v_{\text {es }}(z)\right]$ $=E(z)$. $\}$ Since $E(z)$ was determined in Sec. II and $\delta n(z)$ in Sec. III, we have the information required to determine $V(z)$ according to Eq. (10b). ${ }^{15}$ In Fig. 5, we show graphs of $V(z)$ for three electric field strengths-namely, $E=0,2 \times 10^{7}$, and $10^{8} \mathrm{~V} / \mathrm{cm}$. In addition, we show the potential obtained from assuming a triangular barrier with an image correction, ${ }^{9}$ having the same height as our potential. Notice that this potential barrier is not as wide as our potential and therefore leads to larger emission currents.

With the one-electron potential determined, we now can calculate tunneling currents using the method described by Duke. ${ }^{9}$ Far from the surface, the potential decreases linearly; as is well known, the solutions to the Schrödinger equation in such a potential are the Airy functions $\mathrm{Ai}(z)$ and $\mathrm{Bi}(z)$. We are interested in a solution which has the form of an outgoing wave, so the proper linear combination is $T[\mathrm{Bi}(z)+i A \mathrm{i}(z)]$, where $\tau$ is a constant. We calculated this solution and its derivative at a point far outside the surface and then used these as initial values in a Runge-Kutta integration of the Schrödinger equation into the metal. Deep inside the metal, the resulting solution and its derivative were matched to an incident plus a reflected plane wave, $e^{i k_{z} z}+R e^{-i k_{z^{z}}}$, to determine $R$ and $\tau$. If we let $E_{z}=\hbar^{2} k_{z}^{2} / 2 m$ (i. e., $E_{z}$ is the kinetic energy associated with motion normal to the surface),

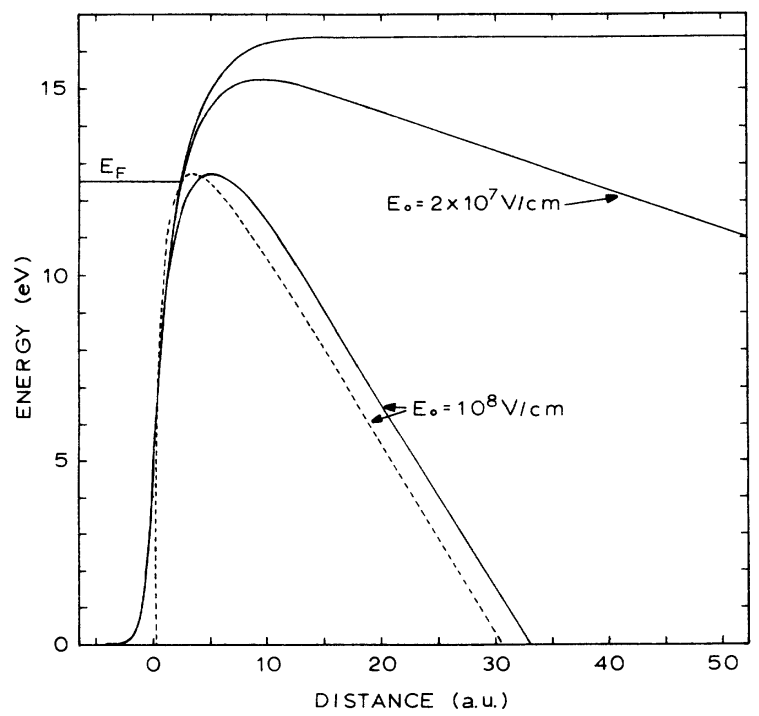

FIG. 5. Dependence of potential upon field strength for $r_{s}=2$. The dashed line is the triangular barrier plus image term for a metal with the work function of a semiinfinite free-electron model with $r_{s}=2 . \quad E_{F}$ is the Fermi energy. The unlabeled solid line corresponds to $E_{0}=0$.

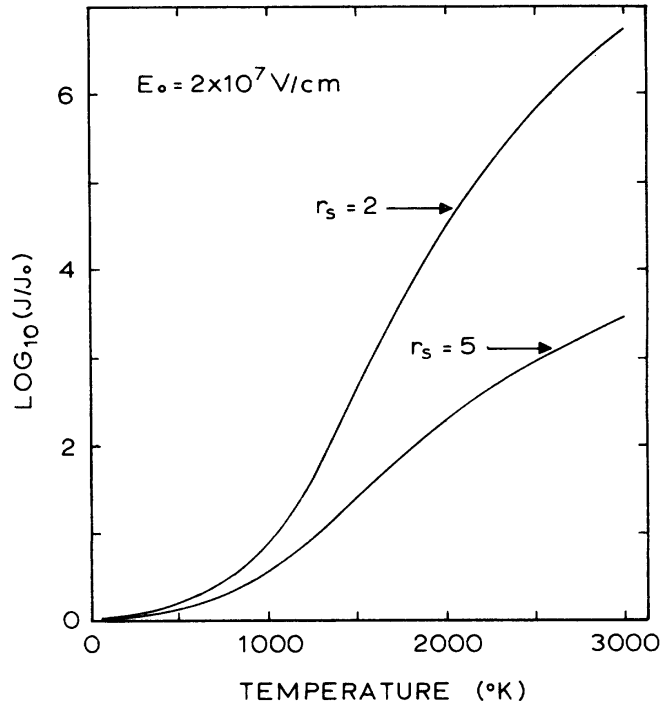

FIG. 6. Dependence of emission current on temperature for a fixed field strength $E_{0}=2 \times 10^{7} \mathrm{~V} / \mathrm{cm}$ and for both $r_{s}=2$ and 5 . The temperature range is $0-3000^{\circ} \mathrm{K}$.

$j_{T}\left(E_{\varepsilon}\right)$ be the probability current outside the metal (which can be determined after the wave function is determined as described above), $j_{0}\left(E_{\varepsilon}\right)$ be the probability current of the incident wave $e^{i k} \varepsilon^{z}$, and $D\left(E_{z}\right)=j_{T}\left(E_{z}\right) / j_{0}\left(E_{\varepsilon}\right)$, then the emission current can be calculated as a function of the temperature $T$ using the result ${ }^{9}$

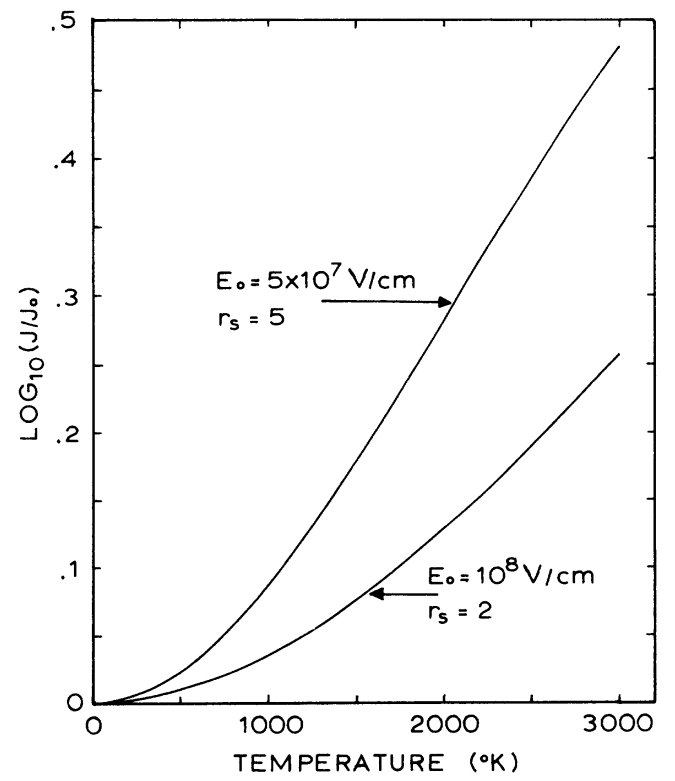

FIG. 7. Dependence of emission current on temperature for fields of $5 \times 10^{7} \mathrm{~V} / \mathrm{cm}$ at $r_{3}=5$ and $10^{8} \mathrm{~V} / \mathrm{cm}$ at $r_{s}=2$. The temperature range is $0-3000 \%$. 


$$
\begin{aligned}
& J(T)=e \int_{0}^{\infty} D\left(E_{z}\right) N\left(E_{z}, T\right) d E_{z}, \\
& N\left(E_{z}, T\right)=4 \pi m\left(k_{B} T / h^{3}\right) \ln \left[1+e^{\left(B_{F}-E_{z}\right) / k_{B} T}\right],
\end{aligned}
$$

where $k_{B}$ is the Boltzmann constant, $m$ is the electronic mass, and $E_{F}$ is the Fermi energy.

In Fig. 6 we show the dependence of the emission current $J(T)$ on temperature for two choices of the electronic density (i.e., $r_{s}$ ) and for temperatures between 0 and $3000{ }^{\circ} \mathrm{K}$, at a field strength of $2 \times 10^{7} \mathrm{~V} / \mathrm{cm}$. In Fig. 7 we show $J(T)$ vs $T$ for stronger fields, i. e., $5 \times 10^{7} \mathrm{~V} / \mathrm{cm}$ for $r_{s}=5$ and $10^{8} \mathrm{~V} / \mathrm{cm}$ for $r_{s}=2$. These currents appear to be in general agreement with, e.g., those of Dolan and Dyke ${ }^{8}$ obtained with the triangular barrier plus an image term.

\section{SUMMARY}

The purpose of this paper was to consider three problems within the context of the self-consistent, semi-infinite free-electron model: First, we determined the screening of an external electric field near a metal surface using a linear-response theory; the results for the screened field $E(z)$ and the induced charge $\delta n(z)$ are shown in Figs. 1-4. Second, we considered the equivalent problem of excess charge in a metal; the distribution of the excess charge $\delta n(z)$ and the electric field $E(z)$ associated with this excess charge are again given in Figs. 1-4. We found that exchange and correlation effects (in a local-density approximation) decrease the effectiveness of the screening, in that the Friedel oscillations in the electric field are enhanced. We also found that the electric field may sometimes be overscreened at the center of the first layer of ions, so that a positive test charge at this point would be attracted by a positive sheet of charge outside the metal. Finally, we calculated field-emission currents which are produced when electrons tunnel quantum mechanically out of the metal in the presence of a strong negative electric field. To our knowledge, these are the first calculations of tunneling currents for a truly selfconsistent potential; the results are somewhat smaller than would be obtained from the triangular barrier plus an image term, since we find that this potential is somewhat less broad than our selfconsistent potential.
*Work supported in part by a grant from the Texas A\&M Research Council.

${ }^{1}$ L. I. Schiff, Phys. Rev. B 1, 4649 (1970).

${ }^{2}$ C. Herring, Phys. Rev. 171, 1361 (1968).

${ }^{3}$ A. K. Theophilou and A. Modinos, Phys. Rev. B 6, 801 (1971).

${ }^{4}$ T. T. Tsong and E. W. Muller, Phys. Rev. 181, 530 (1969).

${ }^{5}$ A. V. Sidyakin, Zh. Eksp. Teor. Fiz. 58, 573 (1970) [Sov. Phys.-JETP 31, 308 (1970)].

${ }^{6}$ R. H. Fowler and L. Nordheim, Proc. R. Soc. A119, 173 (1928)

${ }^{7}$ R. H. Fowler, Proc. R. Soc. A122, 36 (1929).

${ }^{8}$ W. W. Dolan and W. P. Dyke, Phys. Rev. 95, 327 (1954).

${ }^{9}$ C. B. Duke, Tunneling in Solids (Academic, New York, 1969),
${ }^{10}$ N. D. Lang and W. Kohn, Phys. Rev. B 1, 4555 (1970). ${ }^{11}$ V. E. Kenner, R. E. Allen, and W. M. Saslow, Phys. Lett. 38A, 255 (1972).

${ }^{12}$ E. P. Wigner, Phys. Rev. 46, 1002 (1934).

${ }^{13}$ N. D. Lang and W. Kohn, Phys. Rev. B 3, 1215 (1971).

${ }^{14} \mathrm{As} z$ increases beyond the peak, the ratio $\delta n(z) / n_{0}(z)$ increases, but since $\delta n(z)$ itself decreases rapidly there is a relatively small contribution to the screened electric field and the induced potential from the region where $\delta n / n$ is large.

${ }^{15}$ The zero-field potential $v_{0}(z)$ was obtained from Ref. 10 out to a distance $z=2 \pi / k_{F}$ beyond the surface. Beyond this point an exponential falloff was assumed for the charge distribution, and the corresponding potential was then calculated. 